\title{
MiR-362-5p as a novel prognostic predictor of cytogenetically normal acute myeloid leukemia
}

Qiu-Ling Ma ${ }^{1,2,{ }^{\dagger}}$, Jing-Han Wang ${ }^{1,2 \dagger}{ }^{+}$Min Yang ${ }^{1}$, Huan-ping Wang ${ }^{2}$ and Jie Jin ${ }^{1,2^{*}}$

\begin{abstract}
Background: MicroRNAs are of special interest in cancer research and hold significant promise as diagnostic and prognostic biomarkers for malignant disease. MiR-362-5p have been found to exert both oncogenic and tumor suppressive effects depending highly on the cellular context. The aim of this study was to determine whether the expression of miR-362-5p can be served as a prognostic factor for patients with cytogentically normal acute myeloid leukemia (CN-AML).
\end{abstract}

Methods: We enrolled 224 patients with CN-AML and measured the expression of miR-362-5p by quantitative real time PCR analysis. We classified patients into high and low expression based on the median value. The Cox regression analyses were carried out to assess the prognostic significance of miR-362-5p expression in the context of the wellestablished predictors. Additionally, microRNA expression profiling were conducted to identify the biological insights between high and low group.

Results: High expressers had older age. High expressers obtained shorter overall survival in the univariate analysis. The independent prognostic value of miR-362-5p remained in the context of the well-established clinical and cytogenetic predictors. Moreover, the prognostic value of miR-362-5p was also validated in an independent cohort of CN-AML. Notably, numerous oncomiRs were also high expressed in high miR-362-5p group.

Conclusion: High miR-362-5p expression was associated with poorer overall survival implicating the oncogenic function in AML development.

Keywords: miR-362-5p, Acute myeloid leukemia, Gene expression, Prognosis

\section{Background}

Acute myeloid leukemia (AML) is a group of heterogeneous hematologic malignancy with great variability in molecular signatures, genetic phenotype, prognostic behaviors and treatment outcome [1-4]. Although chromosomal abnormalities have been validated as an effective tool for the classification and risk stratification, chromosomal lesions are identified in approximately $50 \%$ of all AML when assessed using conventional banding

\footnotetext{
*Correspondence: jiej0503@zju.edu.cn

${ }^{\dagger}$ Qiu-Ling Ma and Jing-Han Wang contributed equally to this work

1 Department of Hematology, The First Affiliated Hospital, Zhejiang University of Medicine, No. 79 Qingchun Road, 310003 Hangzhou,

Zhejiang, People's Republic of China

Full list of author information is available at the end of the article
}

analysis. In contrast, about $50 \%$ of all AML cases are cytogenetically normal $(\mathrm{CN})$. In order to refine classification for CN-AML patients, molecular diagnosis, such as NPM1, FLT3-ITD, CEBPA mutational analysis, is crucial $[5,6]$. Beside the genes mutations, oncogenes expression also obtained the prognostic significance in CN-AML [4]. For example, low expression of the brain and acute leukemia cytoplasmic (BAALC) and ETS-related gene (ERG) genes have been associated with better outcome in CN-AML patients [7, 8]. Recently, we found that high expression of HIP1 and IDH1 were associated with poor survival in AML [9]. In addition to genetic alterations in protein coding genes, aberrant expression of noncoding RNAs might play an important role in leukemia initiation and outcome prediction [10]. 
MicroRNA (miRNA) is the first class of noncoding RNAs and constitutes about 19-22 nucleotides that is involved in post-transcriptional regulation of genes and play important roles in biological functions such as cell differentiation, proliferation, DNA repair, cell adhesion, motility, and apoptosis [11, 12]. The aberrant expression of miRNAs is identified in various disease and cancer [13]. Numerous studies revealed that some miRNA expression significantly influence apoptosis, block hematopoietic differentiation, induce leukemia transformation and drug resistance by controlling gene expression which associated with diagnosis, prognosis, and response to treatment in leukemia [14].

Notable examples of these miRNAs are given by miR-155, miR-29, miR-150 and miR-196b [15]. Recently, a few studies demonstrated miR-362-5p could act as tumor suppressors by targeting phosphatidylinositol 3-kinase-C2beta and inhibiting proliferation and migration of neuroblastoma cells [16]. On the other hand, miR-362-5p could play an oncogenic role by down-regulating tumor suppressor CYLD in gastric cancer and hepatocellular carcinoma [17, 18]. With respect to hematological malignances, miR-326-5p can promote the malignancy of chronic myeloid leukemia via down-regulation of GADD45alpha [19]. However, the role of miR-362-5p in acute myeloid leukemia remains unclear. Here, we investigate the prognostic value of miR-326-5p in CN-AML patients.

\section{Methods \\ Patients}

In this study, we selected 224 patients with de novo $\mathrm{CN}$-AML in the center of Zhejiang Institute of Hematology (ZIH), Hangzhou, China from 2008 to 2015. All patients were well-informed about the study and provided written informed consent to participate in the study. The study was approved by the Institutional Review boards of our hospital. The research was conducted in accordance with the Helsinki Declaration. Patients with CN-AML were included in this study. Bone marrow (BM) samples were obtained at the time of diagnosis. Mononuclear cells were isolated from the BM samples by Ficoll-Hypaque density-gradient centrifugation and subsequently used for mutational molecular analyses. To be considered cytogenetically normal, at least 20 metaphase cells from diagnostic bone marrow had been evaluated. Patients with $\mathrm{CN}$ AML are treated with standard Anthracycline and Cytarabine. With respect to the consolidation therapy, these patients were treated with a high-dose Cytarabine-based chemotherapy.

\section{Cytogenetic and gene mutation analysis}

Cytogenetic and molecular studies were performed at $\mathrm{ZIH}$. The pretreatment BM samples from all CN-AML patients were studied by R-banding analysis. The definition of a cytogenetic clone and descriptions of karyotypes followed the International System for Human Cytogenetic Nomenclature [20].

DNA and RNA samples obtained from mononuclear cells from BM samples at primary diagnosis were extracted as described previously [9]. Mutation analyses of NPM1, FLT3-ITD, CEBPA, DNMT3A, IDH1 and IDH2 were carried out as described previously [9].

Bone marrow (BM) mononuclear cells (MNCS) were purified by Ficoll density gradient centrifugation and were dissolved by RNAiso plus (Takara, Japan). Total RNA was extracted and purified using miRNeasy Mini Kit (Qiagen, USA) following the manufacturer's instructions. $1 \mu \mathrm{g}$ total RNA was used for cDNA by All-inOneTM miRNA First Strand cDNA Synthesis Kit(Gene Copeia, USA). miRNA q-PCR was conducted using ALL-in-one ${ }^{\mathrm{TM}}$ miRNA qPCR kit (Gene Copeia, USA). The assay was carried out on an IQ5 Real Time PCR instrument (Bio-Rad, USA), PCR reactions were performed in a total volume of $20 \mu \mathrm{l}$ containing of $2 \mu \mathrm{l}$ sample cDNA, $10 \mu \mathrm{l}$ of $2 \times$ All-in-One qPCR Mix, $2 \mu \mathrm{l}$ of All-in-one miRNA qPCR Primer ( $2 \mathrm{uM}), 2 \mu \mathrm{l}$ of Universal Adaport PCR Primer $(2 \mathrm{uM})$, and $4 \mu \mathrm{l}$ of cDNA, RNase/DNase free $\mathrm{H}_{2} \mathrm{O}$. The reactions were incubated in a 96-well plate at $95^{\circ} \mathrm{C}$ for $10 \mathrm{~min}$, followed by 40 cycles of $95^{\circ} \mathrm{C}$ for $10 \mathrm{~s}$, $58{ }^{\circ} \mathrm{C}$ for $40 \mathrm{~s}$ and $72{ }^{\circ} \mathrm{C}$ for $15 \mathrm{~s}$. Relative quantification was calculated using $2^{-\Delta \Delta C T}$ [21] and U6 was used for normalization. The primers used for q-PCR were miR362-5p (5'-ATCCTTGGAACCTAGGTGTGAGT-3') and U6 (5'-TTCGTGAAGCGTTCCATATTTT-3').

\section{MicroRNA experiments}

For miRNA profiling, total RNA was extracted and purified using mirVana ${ }^{\mathrm{TM}}$ miRNA Isolation Kit (Ambion, Austin, TX, US) following the manufacturer's instructions. RNA integrity number (RIN) was assessed by an Agilent Bioanalyzer 2100 (Agilent technologies, Santa Clara, CA, US). miRNA expression was performed using the Agilent Human miRNA Microarray Kit Version 16.0. Total RNA (100 ng) was hybridized per sample and processed according to the manufacturer's instructions. The arrays were scanned by an Agilent Technology G2565BA scanner. The scanned images were gridded and analyzed with Agilent Feature Extraction Software Version 10.7. Raw data were normalized by quantile algorithm, Gene Spring Software 11.0. Each microRNA signature was represented by the average of its expression value of replicate probes. 


\section{Statistical analysis}

The main objective of this study was to evaluate the association between miR-362-5p expression and overall survival in patients with CN-AML. We classified CN-AML patients into high and low groups based on the median value of miR-362-5p expression. Patient characteristics were summarized using descriptive statistics, which included frequency counts, median and range. The relationship between miR-362-5p expression and patient characteristics was estimated by the nonparametric test and Chi square test. OS was measured as time from disease diagnosis to death from any cause, or censoring for patients alive at their last follow-up. Only 4 patients received bone marrow transplantation (BMT) in our patients, and OS of these patients were censored at days for patients with BM transplantation. Kaplan-Meier method used in univariate analysis and Cox proportional hazard regression model in multivariate analysis were used to determine the prognostic value of miR-362-5p expression. All statistical analyses were conducted with $\mathrm{R}$ statistic package, version 3.3.1 (http://www.r-proje ct.org). $P<0.05$ demonstrated statistical difference.

\section{Results}

\section{Clinical characteristics of CN-AML patients with aberrant} expression of miR-362-5p

Clinical characteristics of $224 \mathrm{CN}$-AML patients with high and low miR-362-5p expression were summarized in Table 1. High miR-362-5p expression was predominant in older patients. CN-AML patients with high miR362-5p expression had higher complete remission rate compared to those with low expressers $(67.9 \%$ vs. $56.2 \%$, $P=0.098)$, however, the difference did not reach the statistical significance. There was no statistically significant correlation between miR-362-5p expression and other variables including sex, white blood cell counts (WBC), hemoglobin, platelet counts (PLT), percentage of bone marrow blasts, FAB subtypes and genes of FLT3-ITD, CEBPA, NPM1, DNMT3A, IDH1 and IDH2 mutations (Table 1).

\section{Associations of miR-362-5p expression and overall survival} In this study, the 3-year overall survival (OS) rate of $224 \mathrm{CN}$-AMLs was $50 \%$. We observed 86 death in this cohort, 44 (51\%) death due to no remission, 32 (37\%) death from disease relapse, $6(7 \%)$ death from infection to $4(5 \%)$ death because of cerebral hemorrhage during intensive consolidation treatment As shown in Fig. 1a, high miR-362-5p expressers $(n=112)$ had poorer OS than low expressers $(n=112)$. Notably, miR362-5p expression was as an independent prognostic factor in multivariate analysis for OS after adjusting for
Table 1 Clinical characteristics of patients with aberrant expressed miR-362-5p

\begin{tabular}{|c|c|c|c|}
\hline Variables & Low expression & High expression & $P$ value \\
\hline Number & 112 & 112 & \\
\hline Sex, n (\%) & $60(53.6)$ & $71(63.4)$ & 0.175 \\
\hline Age, years ${ }^{\#}$ & $45.50[33.00,59.00]$ & $50.50[41.25,62.00]$ & 0.048 \\
\hline WBC, $10^{9} /$ I $^{\#}$ & $18.60[4.79,64.65]$ & $28.30[5.80,93.55]$ & 0.301 \\
\hline Hemoglobin, $/\left.\right|^{\#}$ & $79.50[66.00,95.00]$ & $88.50[67.00,105.25]$ & 0.061 \\
\hline $\mathrm{PLT}, 10^{9} / \mathrm{I}^{\#}$ & $38.50[22.75,77.25]$ & $46.50[25.00,109.50]$ & 0.141 \\
\hline Blast, \% ${ }^{\#}$ & $69.00[54.00,79.62]$ & $71.50[48.38,82.62]$ & 0.920 \\
\hline FAB subtype, n (\% & & & 0.904 \\
\hline Mo & $9(8.0)$ & $11(9.8)$ & \\
\hline M1 & $12(10.7)$ & $11(9.8)$ & \\
\hline M2 & $47(42.0)$ & $50(44.6)$ & \\
\hline M4 & $14(12.5)$ & $9(8.0)$ & \\
\hline M5 & $28(25.0)$ & $28(25.0)$ & \\
\hline M6 & $2(1.8)$ & $3(2.7)$ & \\
\hline \multicolumn{4}{|c|}{ Genes mutation, n (\%) } \\
\hline FLT3-ITD & $19(17.0)$ & $24(21.4)$ & 0.498 \\
\hline CEBPA $^{\text {DM\& }}$ & $14(12.5)$ & $17(15.2)$ & 0.699 \\
\hline NPM1 & $21(18.8)$ & $33(29.5)$ & 0.085 \\
\hline DNMT3A & $10(8.9)$ & $12(10.7)$ & 0.823 \\
\hline $\mathrm{IDH} 1$ & $7(6.2)$ & $12(10.7)$ & 0.338 \\
\hline $\mathrm{IDH} 2$ & $11(9.8)$ & $19(17.0)$ & 0.169 \\
\hline CR rates & 76 (67.9) & $63(56.2)$ & 0.098 \\
\hline
\end{tabular}

WBC white blood cell counts, PLT platelet counts, DM\& double allele mutations

\# Median (interquartile)

age, WBC, hemoglobin levels and genes of FLT3-ITD, NPM1, CEBPA, DNMT3A, IDH1 and IDH2 mutations. Additionally, the well-established prognostic factors like age, WBC, and genes of CEBPA and NPM1 mutations were still as the independent prognostic factors in multivariate analysis (Table 2).

In the TCGA dataset, $80 \mathrm{CN}$-AML patients were classified into high and low group based on the median value of miR-362-5p. We found patients in high miR362-5p group had poor overall survival than those in low group (Fig. 1b).

\section{MicroRNA expression profiling}

We used 12 samples with high miR-362-5p expression and 12 samples with low expression to assess the differences of microRNA (miR) expression. The most significant changes of miRs in high expressers included up-regulation of miR-532-3p, miR-362-3p, miR-660-5p, miR-532-5p, miR-210-3p, miR-502-3p, miR-500a-3p, miR-502-5p, miR-660-3p, miR-4633-5p, miR-4501, miR-1247-5p, let-7c-5p, miR-449a and down-regulation of miR-663a, miR-4538, miR-3155b, miR-6500-3p, miR550b-3p, miR-3687 ( $P$ value $<0.005$, Fig. 2 ). 

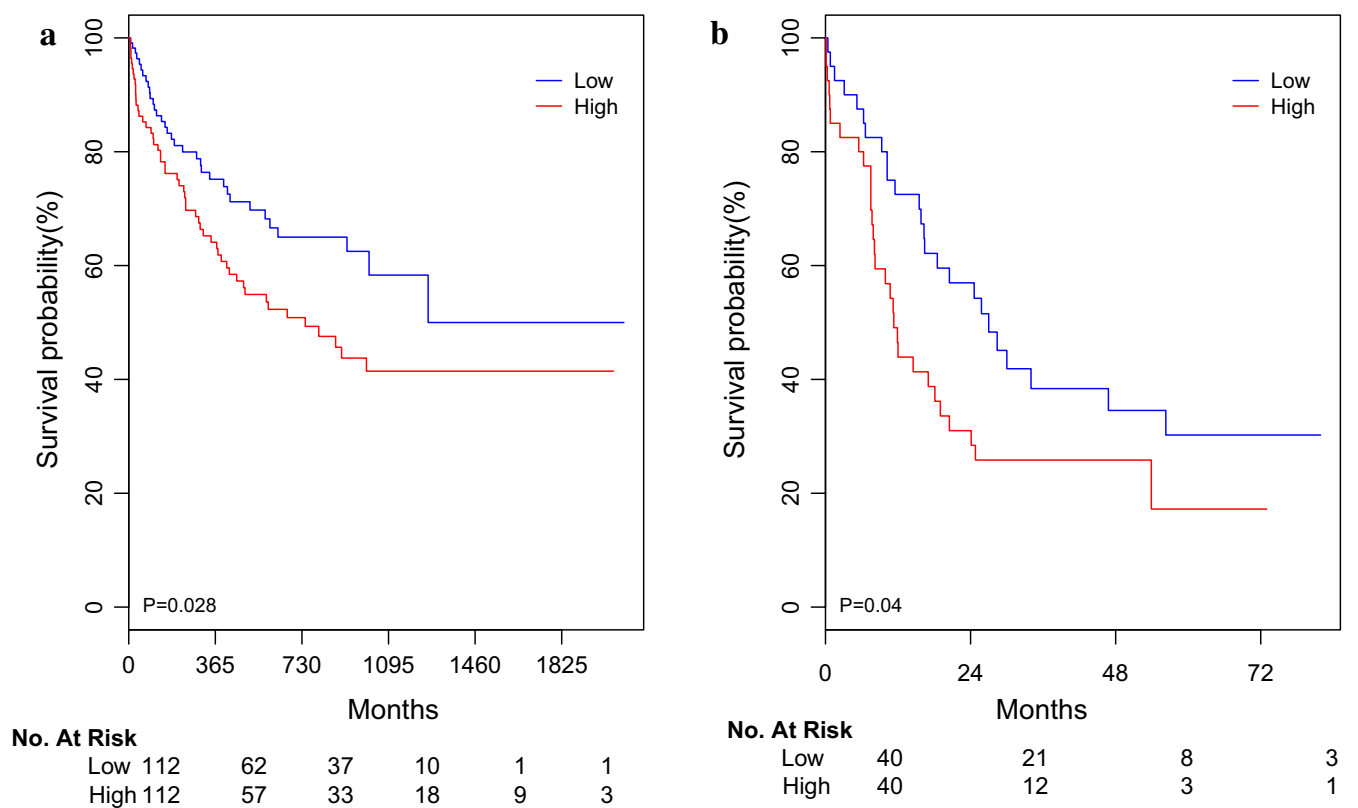

Fig. 1 a Kaplan-Meier survival analysis of the 224 CN-AML patients. OS curves of cases with high or low level of miR-362-5p expression values based on median value of miR-362-5p expression in CN-AML patients. b Kaplan-Meier survival analysis of CN-AML patients as a validation cohort treated according to the TCGA dataset

Table 2 Overall survival analyses for CN-AML patients

\begin{tabular}{|c|c|c|c|c|}
\hline \multirow[t]{2}{*}{ Variables } & \multicolumn{2}{|c|}{ Univariate analysis } & \multicolumn{2}{|c|}{ Multivariate analysis } \\
\hline & $P$ value & HR (95\%) & $P$ value & HR (95\%) \\
\hline miR-36 & 0.028 & $1.615(1.048,2.488)$ & 0.045 & $1.599(1.011,2.528)$ \\
\hline WBC & $<0.001$ & $1.005(1.003,1.008)$ & )$<0.001$ & $1.006(1.003,1.009)$ \\
\hline Hemoglobin & 0.242 & $0.995(0.987,1.003)$ & 0.297 & $0.995(0.987,1.004)$ \\
\hline Age & 0.006 & $1.02(1.006,1.035)$ & 0.005 & $1.022(1.007,1.038)$ \\
\hline FLT3-ITD & 0.654 & $1.136(0.65,1.987)$ & 0.723 & $1.11(0.622,1.982)$ \\
\hline CEBPA $^{\mathrm{DM} \&}$ & 0.014 & $0.352(0.153,0.809)$ & 0.007 & $0.312(0.133,0.732)$ \\
\hline NPM1 & 0.884 & $1.038(0.629,1.712)$ & 0.039 & $0.547(0.309,0.969)$ \\
\hline DNMT3A & 0.157 & $1.58(0.838,2.979)$ & 0.356 & $1.376(0.699,2.711)$ \\
\hline $\mathrm{IDH} 1$ & 0.043 & $1.879(1.019,3.466)$ & 0.125 & $1.662(0.868,3.183)$ \\
\hline $\mathrm{IDH} 2$ & 0.613 & $0.849(0.45,1.601)$ & 0.196 & $0.644(0.331,1.254)$ \\
\hline
\end{tabular}

MiR-362-5p: high vs low expression, Age, WBC and hemoglobin are used as continuous variables

$D M \&$ double allele mutations

\section{Discussion}

AML is a highly heterogeneous disease characterized by failure of terminal differentiation from precursor cells into mature blood cells. miRNAs are small, non-coding RNAs that bind target mRNAs leading to their degradation or disruption of cellular proliferation and differentiation in acute myeloid leukemia (AML) [14]. For example, miR-125 and miR-126 control the PI3K-AKT-mTOR pathway, a gatekeeper of leukemic stem cells self-renewal and transient cell-cycle quiescence [22-24]. Additionally, it has been reported that both single miRNA and panel of miRNAs have prognostic significance in patients with AML. Reduced miR-124-1, miR-125, miR-126 expression had longer survival [14]. In contrast, up-regulated miR320, miR-146, miR-130, miR-223, miR-378 and miR-551b had poor survival in AML [25]. Based on these ground, we believe some miRNAs should act as major players in the development of AMLs and can be used as biomarkers and drug targets.

Recently, miR-362-5p was reported as onco-microRNA in solid tumors $[17,18]$. With respect to hematological malignancy, miR-362-5p expression is higher in both CML patient's samples and cell lines compare to controls [19]. Moreover, the down-regulation of miR362-5p significantly suppresses chronic myeloid leukemia (CML) cell proliferation, enhances cell apoptosis, induces cell cycle arrest, and decreases migration and invasion in vitro, whereas a miR-362-5p inhibitor reduced tumor volume and tumor growth in vivo in a xenograft model [17]. Furthermore, miR-362-5p inhibitor increases the sensitivity of CML cell lines to the agent Ara-c [17]. On the other hand, 5-Aza-2'-deoxycytidine (DAC, an inhibitor of DNA methylation) significantly decreased methylation of miR-362-5p promoter, leading to increasing expression of miR-362-5p in human hepatocellular carcinoma cells [26]. Taken together, these results indicate that miR-362-5p acts as a novel oncogenic miRNA 


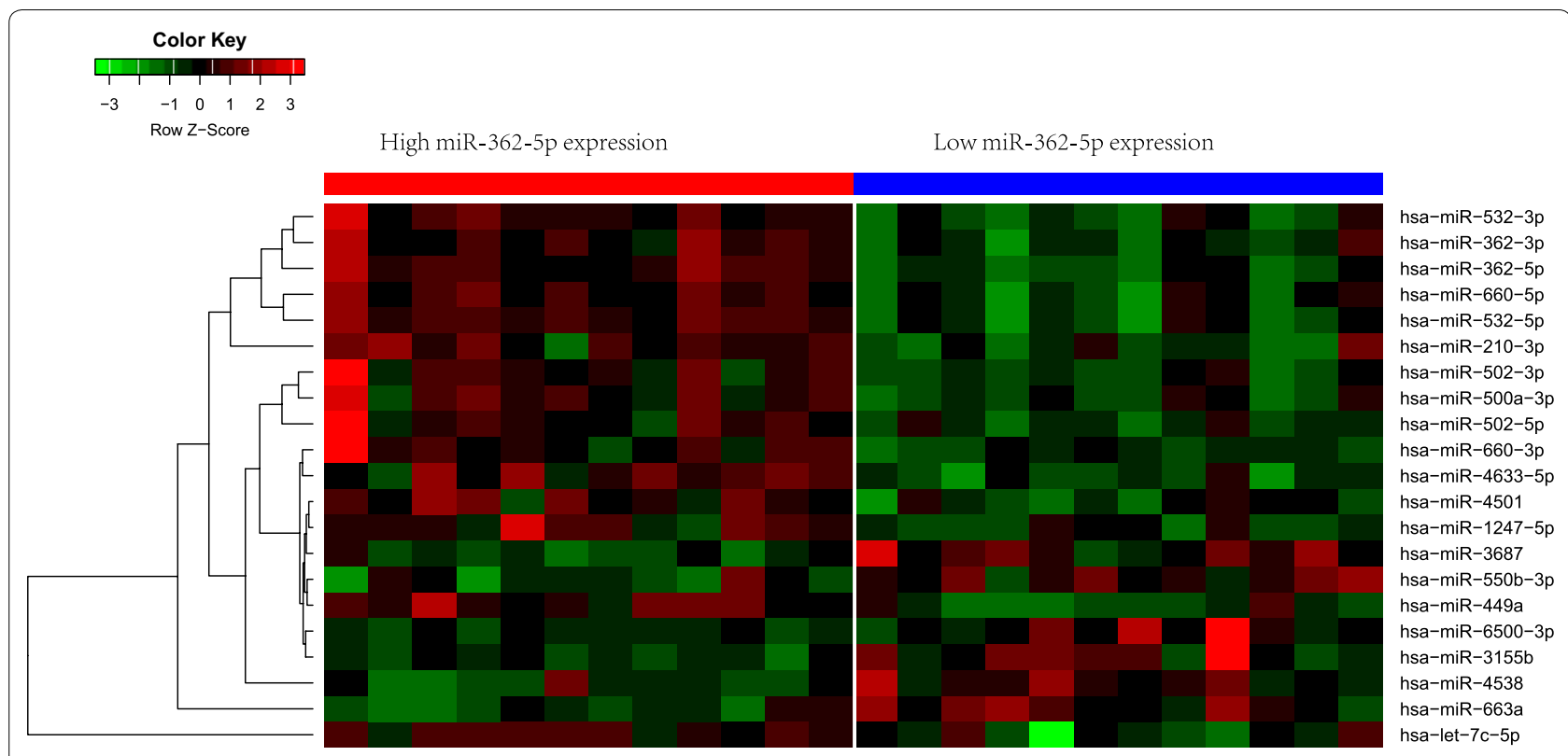

Fig. 2 MicroRNA expression patterns in leukemia blasts of AML patients with high miR-362-5p expression compared to those with low miR-362-5p expression

(oncomiR) that exerts an important effects on solid tumor and leukemia progression. In this study, we examined the expression of miR-362-5p in a large cohort of 224 patients with CN-AML to analyze its prognostic value. In order to test whether it can be used as a reliable prognostic factor, we first classified patients into high and low expressers. We found high expression was predominant in older patients. There were no significant differences between the altered expression of miR-362-5p and the other well-established prognostic factors, such as WBC, hemoglobin and genes of FLT3-ITD, NPM1, CEBPA, DNMT3A, IDH1 and IDH2 mutations. These results indicated that these above factors might not be confounders. When we assumed these well-established factors as potential confounders, we found miR-362-5p expression was still an independent predictor in multivariate models after adjustment of these well-established predictors. Moreover, the prognostic significance was also validated in the TCGA cohort of CN-AMLs. These results indicated that miR-362-5p might act as a reliable and independent predictor in the clinical practice.

It is well known that single miRNA can combine with multiple miRNAs and regulate multiple mRNAs. Therefore, we performed miRNA profiling analysis. As expected, we found two clusters of miRNAs positively and negatively correlated with miR-362-5p expression, respectively. These miRNAs have been proved to be important prognostic markers and novel targets for therapy in cancers. For example, miR-660 expression was used as a good candidate for prognosis prediction in breast cancer [27]. MiR-210 which up-regulated can lead to increased reactive oxygen species as reported by Wei Yang [28]. These clusters of miRNAs are helpful for us to understand the underlying biological insights of miR$362-5 p$ used as a predictors for poor OS.

This study explored the clinical significance of miR362-5p expression in a large cohort of CN-AML patients in China. However, one potential limitation to our study is the study design were retrospective. In addition, we did not take into account several factors including underlying comorbidities and genes mutations like ASXL1, TET2 in multivariate survival analysis although we excluded the well-established factors as the potential confounders. However, this result was also validated in an independent cohort of CN-AML patients.

\section{Conclusion}

In conclusion, present study demonstrated that cytogenetically normal AML (CN-AML) patients with high expression of miR-362-5p are associated with poorer overall survival implicating the oncogenic function in AML development. This study suggests miR-362-5p can be used as an independent poor predictor in patients with CN-AML.

\section{Abbreviations}

AML: acute myeloid leukemia; CN: cytogenetically normal; BM: bone marrow; MNCS: mononuclear cells; BMT: bone marrow transplantation; NPM1: nucleophosmin-1; FLT3-ITD: Fms-like tyrosine kinase 3 gene internal tandem duplication; IDH1: isocitrate dehydrogenase 1; IDH2: isocitrate dehydrogenase 2; DNMT3A: DNA methyltransferase 3A; CEBPA: CCAAT/enhancer-binding 
protein alpha; TET2: the TET methylcytosine dioxygenase 2; HIP1: Huntingtin interacting protein $1 ;$ BAALC: the brain and acute leukemia cytoplasmic; ERG: ETS-related gene; ASXL1: additional sex combs like 1.

\section{Authors' contributions}

QLM, JHW and JJ designed the study. QLM, JHW wrote the manuscript. QLM and JHW performed the experiments and statistical analysis of the data. QLM, MY and HPW performed immunophenotyping and gene marker assays. All authors read and approved the final manuscript.

\section{Author details}

${ }^{1}$ Department of Hematology, The First Affiliated Hospital, Zhejiang University of Medicine, No. 79 Qingchun Road, 310003 Hangzhou, Zhejiang, People's Republic of China. ${ }^{2}$ Key Laboratory of Hematopoietic Malignancies, Hangzhou, Zhejiang Province, People's Republic of China. ${ }^{3}$ Department of Hematology, The Second Affiliated Hospital of Henan University of Traditional Chinese Medicine, Zhengzhou, Henan, People's Republic of China.

\section{Acknowledgements}

This work was supported by National Natural Science Foundation of China grants $(81370643,81470305$, U1404806), the Foundation of Innovation Team for Basic and National Public Health Grand Research Foundation (201202017), University Science and Technology Innovation Talent Support Program of Henan Province (17HASTIT046) and Zhejiang natural fund (Y16H160037). Thanks for the anonymous reviewers and editor for their valuable comments and suggestions that helped improve the quality of our manuscript.

\section{Competing interests}

The authors declare that they have no competing interests.

\section{Availability of data and materials}

Please contact author for data requests.

\section{Consent for publication}

Not applicable.

\section{Ethics approval and consent to participate}

This study was approved by the Institutional Ethics Review Board of the First Affiliated Hospital, College of medicine, Zhejiang University, Zhejiang,China. The reference number is 2017-693.

\section{Publisher's Note}

Springer Nature remains neutral with regard to jurisdictional claims in published maps and institutional affiliations.

\section{Received: 27 January 2018 Accepted: 6 March 2018}

Published online: 14 March 2018

\section{References}

1. Döhner H, Estey EH, Amadori S, et al. Diagnosis and management of acute myeloid leukemia in adults: recommendations from an international expert panel, on behalf of the European LeukemiaNet. Blood. 2010;115:453.

2. Nakao M. Mutations and treatment outcome in cytogenetically normal acute myeloid leukemia. N Engl J Med. 2008;358:1909-18.

3. Schlenk RF, Döhner K, Krauter J, et al. Mutations and treatment outcome in cytogenetically normal acute myeloid leukemia. N Engl J Med. 1909;358:1909-18.

4. Prada-Arismendy J, Arroyave JC, Röthlisberger S. Molecular biomarkers in acute myeloid leukemia. Blood Rev. 2016;31:63.

5. Becker H, Marcucci G, Maharry K, et al. Favorable prognostic impact of NPM1 mutations in older patients with cytogenetically normal de novo acute myeloid leukemia and associated gene-and microRNA-expression signatures: a Cancer and Leukemia Group B study. J Clin Oncol. 2010;28:596.
6. Pastore F, Kling D, Hoster E, et al. Long-term follow-up of cytogenetically normal CEBPA-mutated AML. J Hematol Oncol. 2014;7:55.

7. Metzeler KH, Dufour A, Benthaus T, et al. ERG expression is an independ ent prognostic factor and allows refined risk stratification in cytogenetically normal acute myeloid leukemia: a comprehensive analysis of ERG, MN1, and BAALC transcript levels using oligonucleotide microarrays. J Clin Oncol. 2009;27:5031-8.

8. Schwind S, Marcucci G, Maharry K, et al. BAALC and ERG expression levels are associated with outcome and distinct gene and microRNA expression profiles in older patients with de novo cytogenetically normal acute myeloid leukemia: a Cancer and Leukemia Group B study. Blood. 2010;116:5660-9.

9. Ma Q, Wang J, Wang Y, et al. High IDH1 expression is associated with a poor prognosis in cytogenetically normal acute myeloid leukemia. Int J Cancer. 2014;137:1058-65.

10. Pan JQ, Zhang YQ, Wang JH, et al. IncRNA co-expression network model for the prognostic analysis of acute myeloid leukemia. Int J Mol Med. 2017:93(3):663-71.

11. Leighann Macfarlane PRM. MicroRNA: biogenesis, function and role in cancer. Curr Genomics. 2010;11:537-61.

12. Hata A, Kashima R. Dysregulation of microRNA biogenesis machinery in cancer. Crit Rev Biochem Mol Biol. 2016;51:121.

13. Oliveto $S$, Mancino M, Manfrini N, et al. Role of microRNAs in translation regulation and cancer. World J Biol Chem. 2017;8:45-56.

14. Gordon JEA, Wong JJ, Rasko JEJ. MicroRNAs in myeloid malignancies. $\mathrm{Br}$ J Haematol. 2013;162:162-76.

15. Fernandes Q. MicroRNA: defining a new niche in Leukemia. Blood Rev. 2017;31(3):129-38.

16. Wu K, Yang L, Chen J, et al. miR-362-5p inhibits proliferation and migration of neuroblastoma cells by targeting phosphatidylinositol 3-kinaseC2ß. FEBS Lett. 2015:589:1911-9.

17. Ni F, Zhao H, Cui H, et al. MicroRNA-362-5p promotes tumor growth and metastasis by targeting CYLD in hepatocellular carcinoma. Cancer Lett. 2015;356:809-18.

18. Xia JT, Chen LZ, Jian WH, et al. MicroRNA-362 induces cell proliferation and apoptosis resistance in gastric cancer by activation of NF-KB signaling. J Trans Med. 2014;12:33.

19. Yang P, Ni F, Deng RQ, et al. MiR-362-5p promotes the malignancy of chronic myelocytic leukaemia via down-regulation of GADD45a. Mol Cancer. 2015;14:190.

20. Brothman AR, Persons DL, Shaffer LG. Nomenclature evolution: changes in the ISCN from the 2005 to the 2009 edition. Cytogenet Genome Res. 2009;127:1-4

21. Beillard E, Pallisgaard N, Velden VHJVD, et al. Evaluation of candidate control genes for diagnosis and residual disease detection in leukemic patients using 'real-time' quantitative reverse-transcriptase polymerase chain reaction (RQ-PCR) — a Europe against cancer program. Leukemia. 2003; 17:2474.

22. Tang H, Li RP, Liang P, et al. miR-125a inhibits the migration and invasion of liver cancer cells via suppression of the PI3 K/AKT/mTOR signaling pathway. Oncol Lett. 2015:10:681.

23. Lechman E, Gentner B, Ng SK, et al. miR-126 regulates distinct selfrenewal outcomes in normal and malignant hematopoietic stem cells. Cancer Cell. 2016;29:214.

24. Raffel S, Trumpp A. miR-126 drives quiescence and self-renewal in leukemic stem cells. Cancer Cell. 2016;29:133-5.

25. Hajizamani S, Shahjahani M, Shahrabi S, et al. MicroRNAs as prognostic biomarker and relapse indicator in leukemia. Clin Trans Oncol. 2017;19:951-60.

26. Shen H, Li W, Tian Y, et al. Upregulation of miR-362-3p modulates proliferation and anchorage-independent growth by directly targeting Tob2 in hepatocellular carcinoma. J Cell Biochem. 2015;116:1563-73.

27. Krishnan P, Ghosh S, Wang B, et al. Next generation sequencing profiling identifies miR-574-3p and miR-660-5p as potential novel prognostic markers for breast cancer. BMC Genom. 2015;16:735.

28. Yang W, Wei J, Guo T, et al. Knockdown of miR-210 decreases hypoxic glioma stem cells stemness and radioresistance. Exp Cell Res. 2014;326:22. 